\title{
Grain Refinement in a 304 Type Stainless Steel Caused by Multiple Deformation at $0.5 \mathrm{~T}_{\mathrm{m}}$
}

\author{
Andrey BELYAKOV"), Taku SAKAI and Hiromi MIURA
}

Department of Mechanical Engineering and Intelligent Systems,

The University of Electro-Communications, Chofu, Tokyo 182-8585, Japan

1) On leave from the Institute for Metals Superplasticity Problems, Ufa 450001 , Russia.

\begin{abstract}
Structure evolution and deformation behavior of a 304 type austenitic stainless steel were studied in multiple compression at a temperature of $873 \mathrm{~K}\left(0.5 \mathrm{~T}_{\mathrm{m}}\right)$ under a strain rate of about $10^{-3} \mathrm{~s}^{-1}$. The integrating flow curve shows a maximum at moderate strains around 1.5 followed by a minor strain softening at high cumulative strains above 3 . The structural changes taking place during deformation can be characterized by the evolution of elongated subgrains with their low-to-middle angle boundaries as dense dislocation walls at low to moderate strains. These subgrains become more equiaxed and the subboundary misorientations gradually increase with increase in strain. The volume fraction of the highly misoriented substructure substantially increases upon multiple deformation to high cumulative strains above 3 , finally leading to the development of a fine grained microstructure with an average grain size of about $300 \mathrm{~nm}$. The mechanisms of such structural development as well as the relationship between microstructures and deformation behavior are discussed in details.
\end{abstract}

KEY WORDS: austenitic stainless steel; multiple warm compression; geometrically necessary subboundaries; continuous dynamic recrystallization; strain-induced grain refinement.

\section{Introduction}

Production of fine-grained steels and other materials have some high advantages for the improved mechanical properties. Since the new fine-grained microstructures can be developed by plastic deformation and recrystallization, the studies on grain refinement under thermomechanical processing are of specific practical importance. One of most interesting mechanisms of structural changes is dynamic recrystallization (DRX), which can lead directly to new microstructure formation controlled under hot working ${ }^{1,2)}$. The characteristics of DRX, i.e. dynamic grain size evolved, effect of initial microstructure, etc. have been fairly clarified for hot deformation. It is generally agreed that considerable refinement of microstructure can be achieved by decrease in deformation temperature or increase in strain rate. On the other hand, questions concerning the structural changes taking place at high strains are often complicated by some limited workability of most metallic materials at low-to-moderate temperatures. Recently, several techniques including multiple forging have been proposed to provide very high plastic strains ${ }^{3-6)}$.

The aims of the present work are to study the deformation behavior and the structural changes taking place during severe warm straining for a 304 type austenitic stainless steel. The samples were deformed to high strains above 6 in multiple interrupted compression at $873 \mathrm{~K}\left(0.5 \mathrm{~T}_{\mathrm{m}}\right)$, which is considered as a kind of multiple forging. The dynamic mechanisms of new grain evolution as well as the effect of microstructures developed on the mechanical properties are discussed in details.

\section{Experimental Procedure}

A 304 type austenitic stainless steel (C:0.058, Si:0.7, Mn:0.95, P:0.029, S:0.008, Ni:8.35, Cr:18.09, Cu:0.15, Mo:0.13, all in mass \%, and the balance $\mathrm{Fe}$ ) with an initial grain size of about $25 \mu \mathrm{m}$ was used as the samples for multiple compressions. The samples were machined in a rectangular shape with the starting dimension of 9.8:8.0:6.5 mm. Such ratio of about 1.5:1.22:1.0 was kept as a constant through subsequent compression to a strain of 0.4 in each pass. Multiple compression tests were carried out with consequent changing of the loading direction in $90^{\circ}$ through three of mutually perpendicular axes with pass-to-pass. The samples were compressed in vacuum with a powder of boron nitride as a lubricant at $873 \mathrm{~K}\left(0.5 \mathrm{~T}_{\mathrm{m}}\right)$ under a strain rate of about $10^{-3} \mathrm{~s}^{-1}$. The deformed samples were quenched in water, slightly ground to right-angle shape, and then reheated to $873 \mathrm{~K}$ within 0.6 to $0.8 \mathrm{ks}$ in each deformation pass. Metallographic analyses were carried out using an optical microscope and a JEM-2000FX transmission electron microscope on the sections parallel to the compression 
axis. Misorientations of strain-induced (sub)grain boundaries were studied using a conventional Kikuchiline technique with accuracy within one degree. The total number of boundaries was $80 \pm 20$ at each strain level studied.

\section{Results}

The integrating flow curve plotted over sixteen compression passes in Fig. 1 is similar in appearance to that controlled by dynamic recovery; namely, stresses rapidly increase in about three times by first compression and approach a saturation value by subsequent repeated deformation. Strain hardening taking place in each pass becomes quite small after third compression. It is remarkable in Fig. 1 that the yield stresses at reloading are quite different from the one at first pass, i.e. for an annealed state, and, in contrast, roughly similar to the stresses immediately before unloading after second pass. Such behavior may suggest that any static annealing effect hardly took place for a period of reheating time under multiple tests at $0.5 \mathrm{~T}_{\mathrm{m}}$.

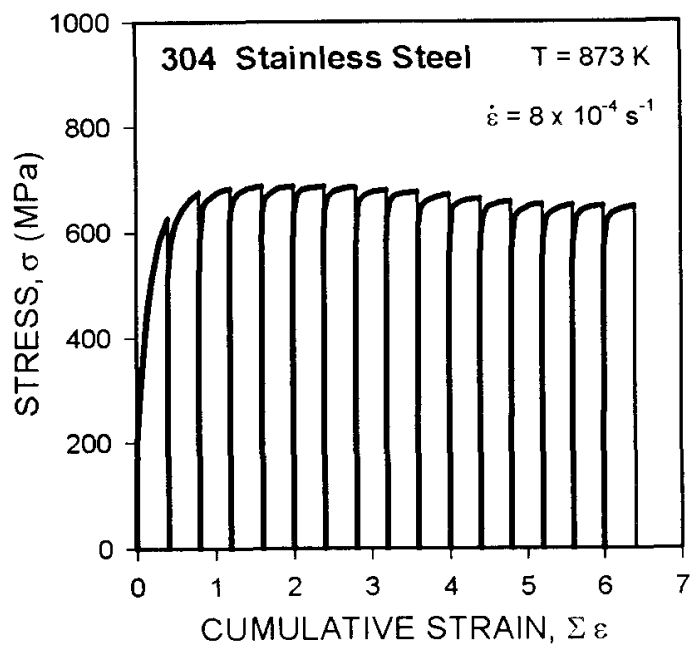

Fig. 1 True stress - cumulative strain curves for 304 stainless steel under multiple compression at $873 \mathrm{~K}$ and at a strain rate of about $10^{-3} \mathrm{~s}^{-1}$

Typical microstructures evolved under warm multiple deformation to various strains are shown in Fig. 2. Structural changes can be characterized by the development of curly microstructure composed of frequently serrated grain boundaries at moderate strains above 1 following pancaked grains evolved by early deformation. With further deformation to high strains, the microstructural analysis becomes more complicated because of high density of irregular grain boundaries as well as deformation bands. It should be especially noted in Fig. 2c that many very fine grains with size of below $1 \mu \mathrm{m}$ can be recognized to be evolved by severe warm deformation.

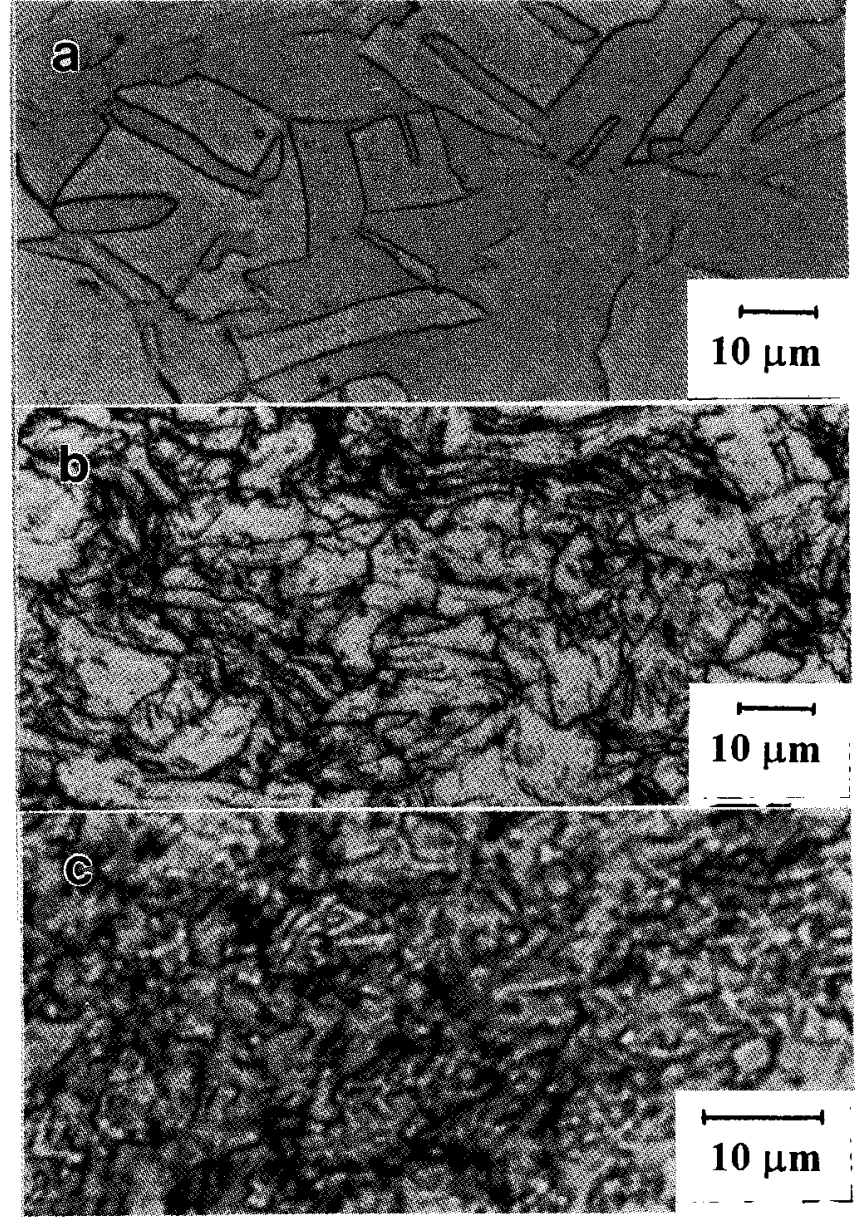

Fig. 2 Typical microstructures developed in 304 stainless steel under warm multiple deformation to various cumulative strains. (a) $\sum \varepsilon=0.4$, (b) $\sum \varepsilon=1.6$ and (c) $\sum \varepsilon=3.2$

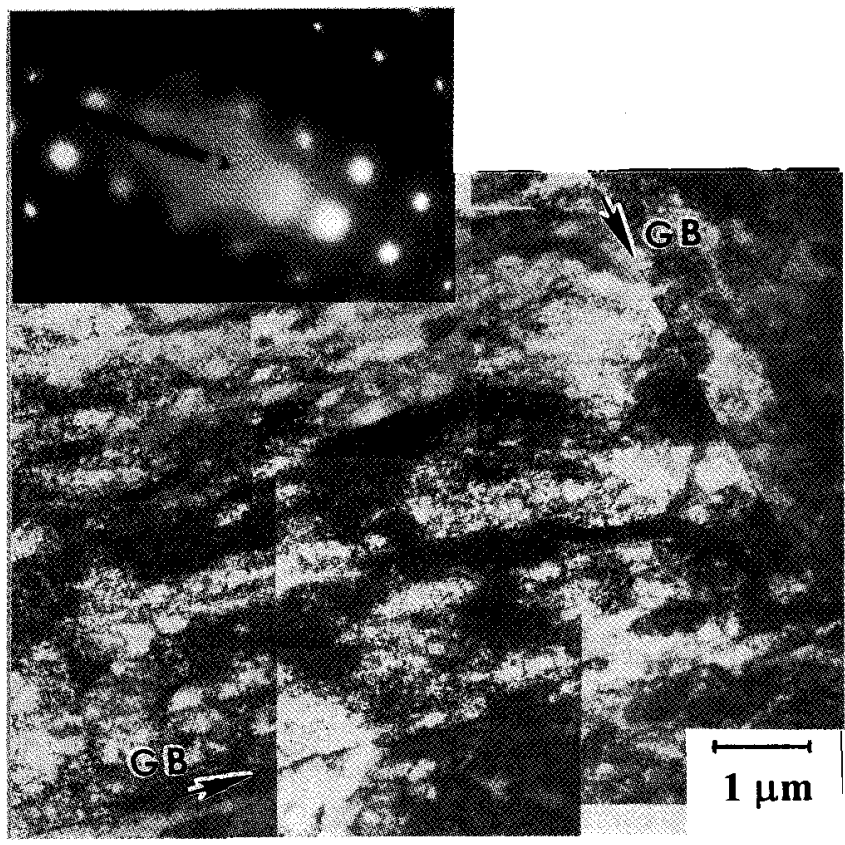

Fig. 3 Cell substructure with high density dislocations in rich dislocation layers near an initial grain boundaries (GB) at $\sum \varepsilon=0.4$. 


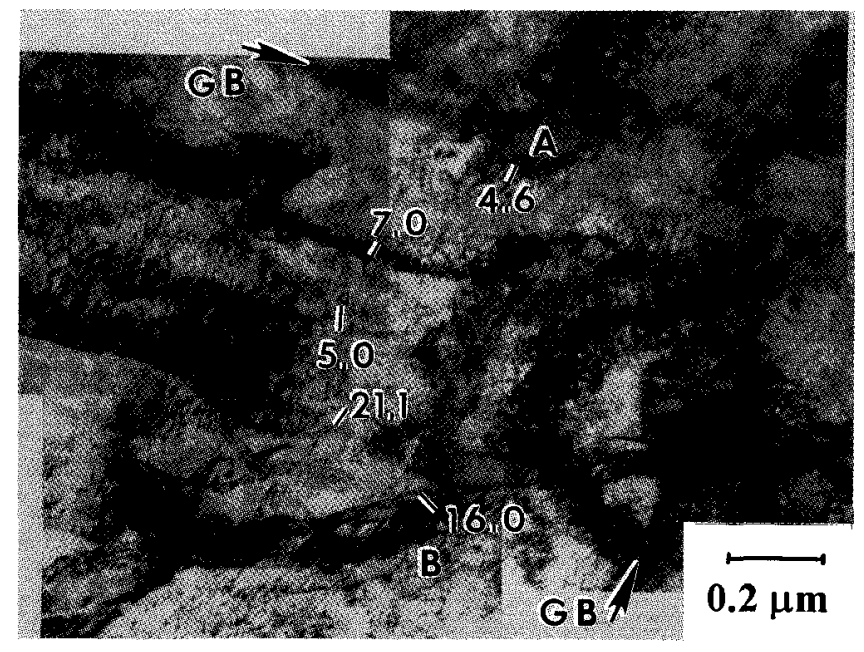

Fig. 4 Strain-induced subboundaries developed in vicinity of a junction of grain boundaries (GB) in 304 stainless steel during warm multiple deformation to $\sum \varepsilon=0.8$. The numbers indicate the misorientation in degrees. Note, the misorientation between points lettered by $\mathrm{A}$ and $\mathrm{B}$ was about $35^{\circ}$.

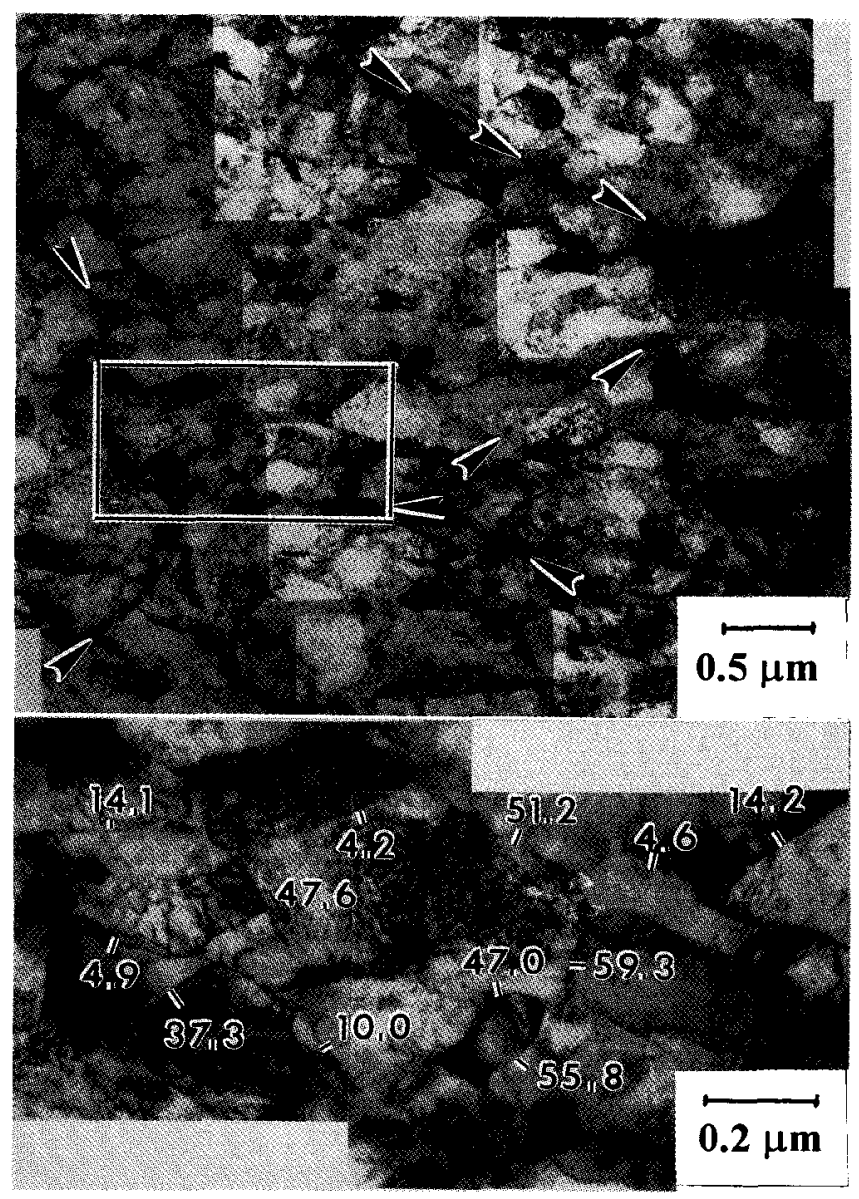

Fig. 5 Typical substructures developed in 304 stainless steel deformed to $\sum \varepsilon=3.2$. The secondary phase precipitations marked by arrow heads may roughly indicate position of initial grain boundaries.
Fig.3 presents a typical substructure evolved at an early stage of deformation corresponding to rapid work hardening. The first compression to a strain of 0.4 leads to the evolution of high density dislocations which are roughly homogeneously arranged in rich dislocation layers. The misorientations across such dislocation subboundaries are about $1-3^{\circ}$, as may be suggested by single spots diffraction pattern obtained from selected area of $6 \mu \mathrm{m}$ in diameter. Further deformation brings about the evolution of dense dislocation walls (DDWs) with low-tomiddle angle misorientations. It can be clearly seen in Fig.4 that the subboundaries with medium to high angle misorientations are frequently formed near the initial grain corner. An another important point to be noted in Fig. 4 is high lattice curvatures and/or rotations developed at the grain corner under warm multiple deformation. The misorientation between points lettered as " $A$ " and " $B$ " was about $35^{\circ}$. This suggests that the grain boundary junctions and ledges should be considered as preferable potential nucleation sites for strain-induced high angle grain boundaries.

A typical microstructure evolved at $\varepsilon=3.2$ is represented in Fig.5. Further multiple deformation to cumulative strain above 3 leads to full development of well defined subgrains with middle-to-high angle boundaries throughout the initial grain interiors. The second phase precipitations, mainly $\sigma$-phase indicated by arrow heads in Fig.5, may roughly indicate the original position of initial grain boundaries, however, the latters cannot be exactly recognized because of the development of many fine grains with ligh-angle boundaries. It is clearly seen in the enlarged lower picture that some of such strain-induced fine grains and/or subgrains contain quite few dislocations and their boundaries give a sharp extinction contrast on TEM image. The latter is typical of conventional grain boundaries.

Upon further multiple compression to severe strains these new fine grains become more equiaxed due to increase in the transverse size and their boundary misorientations gradually increase. The microstructures at $\varepsilon=6.4$ in Fig. 6 can be generally described as an equiaxed grained one with high angle misorientations. The average grain size is about $0.3 \mu \mathrm{m}$. It should also be noted in Fig. 6 that the secondary precipitations are randomly distributed in (sub)grain interiors. This suggests that grain boundary sliding and its local minor migration can take place not only at original grain boundaries but also new straininduced (sub)grain ones

\section{Discussion}

An analysis of the results mentioned above revealed that the new fine grain formation is a consequence of some continuous reactions on substructural level caused by warm multiple deformation. Early deformation brings about high density dislocations followed by the evolution of strain-induced dislocation subboundaries as DDWs with low-to-middle angle misorientations at moderate strains. Gradual rise of the misorientations between subgrains, finally, leads to the full evolution of a new fine- 


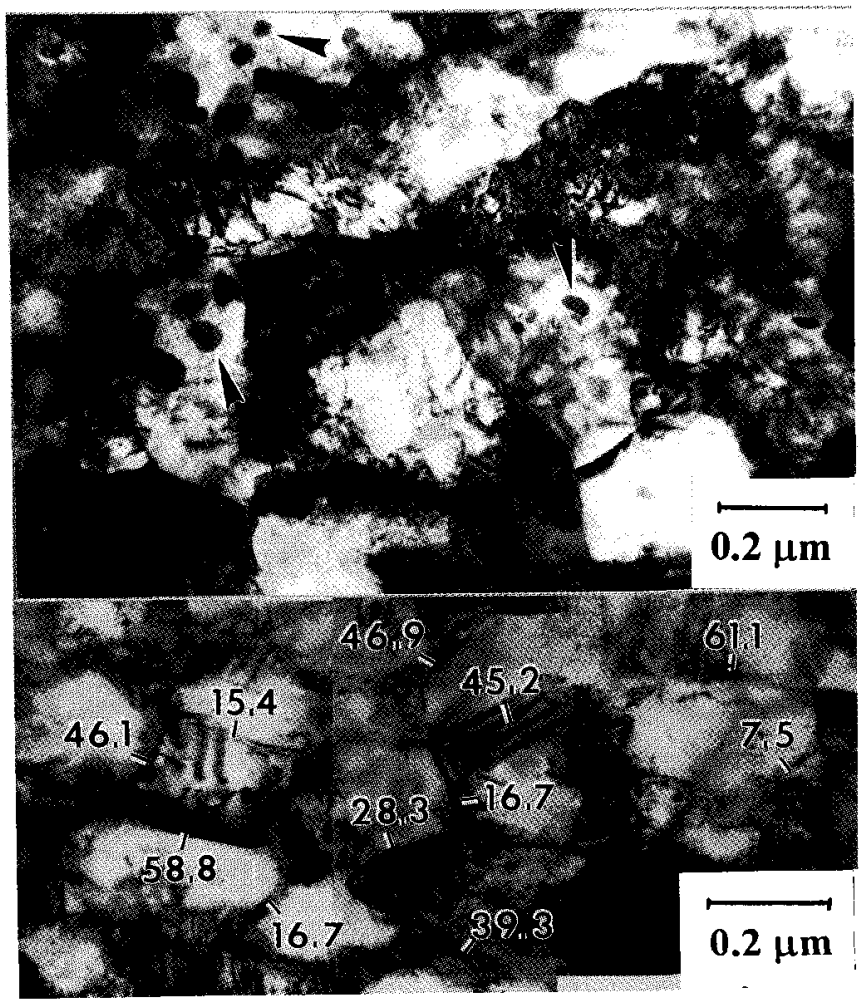

Fig. 6 TEM micrographs of fine-grained microstructures evolved in 304 stainless steel deformed to $\Sigma \varepsilon=6.4$.

grained structure at severe strains assisted by (sub)boundary sliding and recovery. Let us consider the mechanisms of new fine grain formation in some more details.

It is known ${ }^{7,8)}$ that severe plastic deformation can break up the initial grains into domains with different combinations of operative slip systems and, as a geometrical requirement, leads to the evolution of geometrically necessary boundaries (GNBs) between different domains. Multiple compression with changing of the loading directions can accelerate the evolution of many GNBs mutually crossed due to promotion of the variations in slip systems operating at each deformation pass.

By the way, DDWs with relatively high misorientations appears at grain boundary junctions at moderate strains above 1.0 and may also be considered as GNBs, as shown in Fig.4. Such GNBs are frequently evolved near grain boundaries and grain corners to accommodate strain gradients and/or lattice rotations (Fig.4). The latters may be caused by usual strain incompatibilities as well as grain boundary sliding. Such elastic stresses (and local lattice rotations) evolved at grain boundary junctions and ledges can be illustrated by simplified schemes in Fig.7. The sliding or shearing along boundaries marked by " $A$ " and "B" in Fig.7a should lead to the generation of misfit (i.e. virtual) dislocations at the boundary junction because of geometrical differences in the Burgers vectors operating at different boundaries. The accumulation of such misfit dislocations can locally create high elastic lattice distortions which may be similar to those produced by a super dislocation. The high lattice distortion can be also developed at a boundary ledge by usual intrinsic slip because of a difference in misorientation increments at different facets (Fig. 7b). The misfit dislocations at " $B$ " facet in Fig. 7 b may result in local boundary sliding, while those at " $A$ " facet should additionally lead to local lattice rotation, resulting in the evolution of high internal stresses at boundary kinks. Then new GNBs may be developed by secondary slips to decrease the elastic stresses ${ }^{9}$.

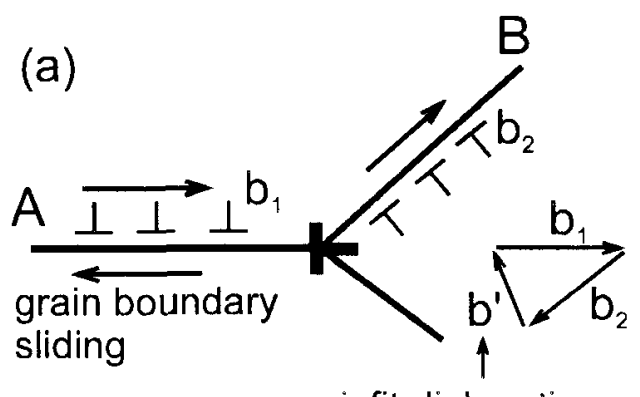

misfit dislocation

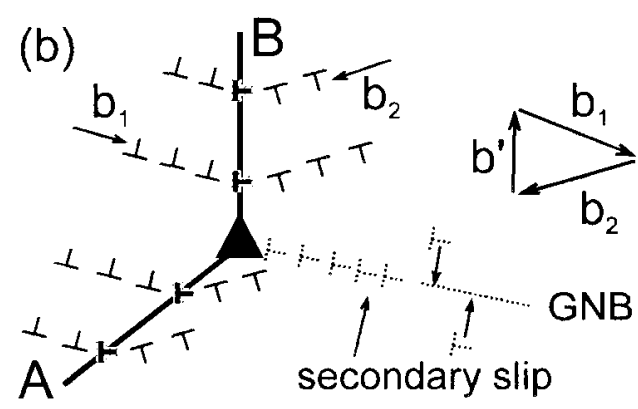

Fig. 7 Long range misfit stresses evolved at boundary junction caused by (a) grain boundary sliding and (b) usual intrinsic slip.

Further straining can cause the number of GNBs and their misorientations to increase. These subboundary misorientations rise due to the increase in the number of lattice dislocations evolved by usual intrinsic slip and the absorption of accommodation (or grain boundary) dislocations that come from strain incompatibility of subgrains. Strain dependence of the development of the strain-induced subboundaries, however, is still under discussions ${ }^{10)}$. The average misorientation of straininduced subboundaries for the present 304 stainless steel linearly increases with low-to-moderate strain, approaching a value of conventional grain boundaries at strains above 3, as shown in Fig.8. In the strain range of 0.5 to 3.0 , the average misorientation $(\theta)$ can be approximated by a linear function of cumulative strain $(\Sigma \varepsilon)$ as

$$
\theta=7(\Sigma \varepsilon-0.2)
$$


This result is quite similar to that reported for a pure copper deformed at $0.35 \mathrm{~T}_{\mathrm{m}}{ }^{\text {) }}$. It should be noted in Fig.8 that a saturation value for the misorientations at high strains is much lower than the theoretical one of $40.7^{\circ} 11$ ). This may be the reasons why such strain-induced grain structure should always contain low angle dislocation subboundaries, which are dynamically evolved during multiple deformation.

It should be noted that the new grains developed at high strains become more equiaxed as compared to preceding subgrains at moderate strains. This may result from local minor migration of strain-induced subboundaries, which in turn should be affected by recovery processes through the promotion of dislocation rearrangement in subboundaries. The phenomenon of new grain evolution described above is similar to continuous and/or rotation DRX taking place in some materials under hot deformation ${ }^{2,12)}$, although the role of dynamic recovery becomes less effective at a relatively low temperature.

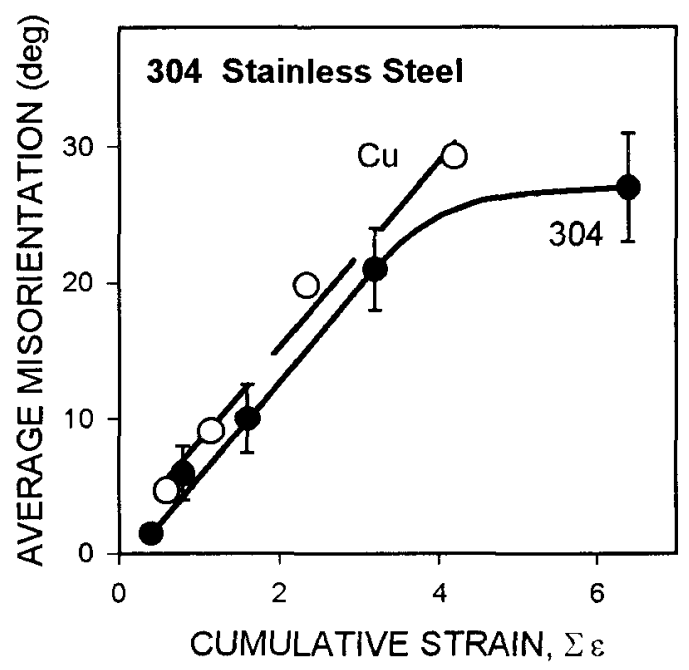

Fig. 8 Variations in average misorientation for straininduced subboundaries with cumulative strain for 304 stainless steel and for $\mathrm{Cu}^{5}$.

\section{Conclusions}

Warm deformation behavior and strain induced grain evolution in 304 type austenitic stainless steel were studied in multipass compression at a temperature of 873 $\mathrm{K}\left(0.5 \mathrm{~T}_{\mathrm{m}}\right)$.

(1) The integrating flow curve increases to a maximum at cumulative strains of about 1.5 followed by a minor softening, leading to a steady-state-like flow at high strains. This suggests that deformation can be controlled mainly by dynamic recovery.

(2) The structural changes are characterized by the evolution of elongated subgrains with their dense dislocation boundaries at low to moderate strains. The subgrains become more equiaxed and the misorientations between them gradually increase with increase in cumulative strain, finally leading to the full development of a new fine-grained structure with an average grain size of about $300 \mathrm{~nm}$.

(3) The fine grain formation at severe high strains can result from a kind of strain-induced continuous reactions taking place during deformation, that is essentially similar to continuous dynamic recrystallization.

\section{Acknowledgments}

The authors acknowledge with gratitude the financial support received from the Recrystallization and Texture Committee of the Iron and Steel Institute of Japan. They also wish to thank the NKK Co. Ltd., for supplying the test material. One of authors (A.B.) also would like to express his hearty thanks to the Inoue Foundation for Science and the University of Electro-Communications for providing scientific fellowships.

\section{REFERENCES}

1) T. Sakai and J. J. Jonas: Acta Metall, 32 (1984), 189

2) F. J. Humphreys and M. Hatherly: Recrystallization and Related Annealing Phenomena, Pergamon Press, Oxford, (1996), 363.

3) N. A. Smimova, V. I. Levit, V. I. Pilyugin, R. I. Kuznetsov, L. S. Davydova and V. A. Sazonova: Phys. Met. Metallogr., 61 (1986), 127.

4) M. Furukawa, Z. Horita, M. Nemoto, R. Z. Valiev and T. G. Langdon: Acta Mater, 44 (1996), 4619.

5) A. Belyakov, W. Gao, H. Miura and T. Sakai: Metall. Trans.A, 29A (1998), 2957.

6) A. Belyakov, T. Sakai, H. Miura and R. Kaibyshev: ISIJ Int., 39 (1999), 592.

7) D. Kuhlmann-Wilsdorf and N. Hansen: Scripta Metall. Mater., 25 (1991), 1557

8) D. A. Hughes and N. Hansen: Acta Mater., 45 (1997), 3871.

9) V. V. Rybin: Large Plastic Deformation and Destructure of Metals, Metallurgy, Moscow, (1986), 61

10) A. S. Argon and P. Haasen: Acta Metall. Mater., 41 (1993), 3289.

11) J. K. Mackenzie: Bionetrika, 45 (1958), 229.

12) T. Sakai, X. Yang and H. Miura: Mater. Sci. Eng., A234-236 (1997), 857 